Apidologie, 1971, 2 (2), 169-172.

\title{
GIBT ES EINE PRÄIMAGINALE INFEKTION BEI DER AMÖBENERKRANKUNG DER HONIGBIENE ?
}

Existe-t-il une infection pré-imaginale dans l'amibiase des abeilles?

\author{
Elisabeth SCHULZ-LANGNER
}

Abt. Bienenkunde des Instituts für Tierzüchtung und Haustiergenetik der Technischen Universität, Berlin

\section{SUMMARY}

COULD AMOEBIASIS

OF THE ADULT BeE RESUlt FRom A PRE-imaginal infection?

A recent publication gave information about MaLPIghamoEBa being present in the Malpighian tubules of newly emerged bees. However, the result of the present investigation turned out to be negative.

\section{ZUSAMMENFASSUNG}

In der neueren Literatur wurde ein Befund von Amöben in den Malpighischen Gefässen frisch geschlüpfter Bienen mitgeteilt. Die vorliegende Untersuchung der Möglichkeit einer prä-imaginalen Infektion verlief jedoch negativ.

Die Bienenamöbe (Malpighamöba mellificae PReLL) ist ein Parasit der Imago. Die Frage, ob die Infektion schon im Larvenzustand übertragen werden kann, ist bisher nie gestellt worden. 1966 berichtet BitTNER zum ersten Mal von Amöbenfunden in Jungbienen und sogar in frisch geschlüpften Tieren. Ein Foto (Abb. 2, S. 285) zeigt in einem Totalpräparat der Malpighischen Gefäße lichtbrechende kugelige Gebilde. Die Anwesenheit von Amöben in 
Harnschläuchen frisch geschlüpfter Bienen setzt voraus, daß die Kontamination der Larven vor dem Verdeckeln stattgefunden hat.

Wenn ich auch der Meinung bin, daß es sich auf dem genannten Foto nicht um abgekugelte Amöbenstadien handelt, läßt sich ein Beherbergen des Parasiten aufgrund der anatomischen Verhältnisse nicht mit Sicherheit ausschließen.

Nach Lotmar (1945) beginnt das Wachstum der imaginalen Malpighischen Gefäße zu kleinen Schläuchen kurz nach der Auflösung des larvalen Mitteldarmverschlusses, die nach Oertel (1930), cit. nach SNodgrass, fünf bis zehn Stunden nach dem Verdeckeln stattfinden soll. Zur gleichen Zeit öffnen sich die bis dahin an ihrer Basis verschlossenen larvalen Malpighischen Gefäße, um ihren Inhalt in das Darmlumen zu entleeren. Die Knospen der imaginalen Malpighischen Gefäße erscheinen unterhalb der Ansatzstelle der larvalen schon etwa fünf Stunden vor dem Verdeckeln (Oentel, 1930), also bereits in der Rundmade. Bei einer 12 Stunden verdeckelten Larve sind die imaginalen Malpighischen Gefäße schon zu kurzen Schläuchen ausgewachsen. Ob sie zum Darmlumen hin wie die larvalen zunächst geschlossen sind, fand ich nirgends erwähnt. In Lotmars Abbildung 1 b, S. 448, zeigen sie in dem frühen Stadium des Diaphragmadurchbruchs im Querschnitt normalgroße Lumina in unmittelbarer Nähe der Mündung. (Längsschnitte mit Mündungsstelle der imaginalen Gefäße sind in dem abgebildeten Präparat nicht getroffen.) Im Falle der offenen Verbindung wäre eine Einwanderung vegetativer Amöbenstadien anatomisch nicht ausgeschlossen, vorausgesetzt, daß das Infektionsmaterial im Larvenmilieu überhaupt keimt. Die im Mitteldarm aufgestauten Pollenmassen gelangen nach Auflösung des Gewebepfropfes nur allmählich in den Hinterdarm; wären vegetative Stadien der Amöbe vorhanden, hätten sie Zeit, den Weg zu den imaginalen Malpighischen Gefäßen zu finden; die Bienenamöbe legt in vitro 1 Millimeter kriechend in 100 Sekunden zurück (Schulz-Langner, 1964).

Die theoretisch nicht abzuweisende Frage, ob die Erkrankung der Imago auch schon aus einer Infektion der Larve stammen kann, wurde in 4 Versuchsanordnungen geprüft :

1. Schlüpfreife Bienen wurden aus dem kranken Völkchen zur Zeit, als die Zahl der Parasitenträger bei über $80 \%$ lag, aus den festverschlossenen Zellen herausgeholt und ihre Malpighischen Gefäße unter dem Mikroskop untersucht. Unter 20 Tieren wurden keine Anzeichen einer Infektion festgestellt. Harnschläuche, die ähnliche Gebilde wie in der Abbildung von Bittrer aufwiesen, wurden auf Nährböden gebracht. Es entwickelten sich keine Amöben.

2. 50 Larven im Alter von 3,5 und 4,5 Tagen wurden in ihren Zellen mit einer Cystenaufsehwemmung in Honig gefüttert. (ca. 10000 Cysten pro $\mathbf{m m}^{3}$ ). Der Honig war mit Pyronin angefärbt, um die Aufnahme in den Darmtrakt augenfällig zu machen. Sämtliche Larven bewiesen mit ihrem rot durchschim- 
mernden Darm, daß sie den Infektionsstoff aufgenommen hatten. Die konsumierte Menge wurde nicht exakt nachgeprüft; der einzige Weg der genauen Erfassung wäre das direkte Füttern der Larven mit der Pipette gewesen. Dieser Aufwand erschien mir überflüssig.

Wie zu erwarten, wurde ein Teil der Larven kurze Zeit später von den Ammenbienen entfernt. Die zur Entwicklung gekommenen 35 Imagines wurden kurz vor dem Schlüpfen aus der Zelle geholt und wie oben untersucht. Ergebnis : Ohne Befund.

3. Die wie in 2.) mit Infektionsstoff gefütterten Larven wurden in kleinen Wachszylindern zur Metamorphose gebracht. An beiden Enden wurden die Röhrchen mit kleinen Deckgläsern verschlossen, um auf diese Weise möglichst den gesamten Larvenkot sauber zu erhalten. Eine Ideallösung war es noch nicht, da die Larven den Kot zum Teil in der Kante zwischen "Zellwand " und Deckglasboden absetzten. Die Kotmassen wurden in Wasser verdünnt auf Objektträger verteilt. Die Untersuchung auf Cysten wurde durch Pollen und Pollenreste sehr erschwert, d. h. es wurden verhältnismäßig wenig Cysten wiedergefunden. $\mathrm{Ob}$ sie der Erfassung entgangen sind oder sich tatsächlich vermindert haben, - sei es durch Zerstörtwerden oder Keimung — bleibt bei dieser Untersuchungsmethode unentschieden. Von 12 Larven machten alle die 5. Häutung durch. Acht Tieren gelang auch die 6. Häutung zur Imago. Sie wurden in kleinen Versuchskäfigen gehalten und blieben erstaunlich lange am Leben (27 bis 38 Tage). Nach ihrem Tod wurden sie untersucht und ebenfalls als amöbenfrei befunden.

4. Eine Wabe mit kleinem Brutnest aus einem amöbenkranken Volk wurde im Thermostaten isoliert. Es sollten ihr Bienen kurz vor dem Schlüpfen, bevor sie also mit dem Infektionsstoff der Wabe durch fütternde Bienen in Berührung gekommen waren, entnommen und auf sterilen Kunststoffwaben gehalten werden. Leider starben die vorzeitig aus den Zellen geholten Tiere schon nach 1-6 Tagen. Amöbencysten wurden nicht gefunden.

Die auf der gleichen Wabe selbständig geschlüpften Tiere wurden ebenfalls in kleinen Kästchen gehalten. Durch ein technisches Versagen gingen sie sämtlich nach 22 Tagen zugrunde. Es wurden keine Amöbencysten gefunden. Die Zeit hätte ausgereicht, um vegetative Stadien zur Cystenreife zu bringen. Auch hier ließ sich keine aus dem larvalen Leben ins imaginale übernommene Infektion nachweisen. (Hätten sich Cysten gefunden, so wäre der Versuch nicht verwertbar gewesen, da die Ansteckung gleich nach dem Schlüpfen hätte erfolgt sein können.)

Nach den vorliegenden Ergebnissen dürfte eine präimaginale Infektion unwahrscheinlich sein. 


\section{RÉSUMÉ}

On a signalé dans la littérature récente un cas de présence d'amibes dans les tubes de Malpighi d'abeilles fraîchement écloses.

Sur la base des faits anatomiques décrits et qui s'appuient sur les travaux de OERTEL (1930) et de Lotmar (1945), et lorsqu'on présume une germination des kystes qui entrent lors de l'alimentation dans l'intestin de la larve, une infection transportée à l'imago pendant la métamorphose n'est pas à exclure a priori.

Cependant le présent travail de recherche sur la possibilité d'une infection pré-imaginale a donné des résultats négatifs.

Au moyen de quatre expériences on a recherché si de telles infections pré-imaginales se produisent effectivement.

1. Des abeilles prêtes à éclore provenant de colonies fortement infectées furent retirées des cellules encore operculées et leurs tubes de Malpighi furent examinés sous le microscope et dans des milieux de culture. Aucune amibe n'a été mise en évidence.

2. 50 larves âgées de 3,5 à 4,5 jours furent alimentées avec du miel contenant une suspension de kystes amibiens. Après l'éclosion les tubes de Malpighi furent examinés comme il est dit au paragraphe 1. Aucun kyste amibien n'a été mis en évidence.

3. Des larves, infectées comme il est dit en 2 furent portées pour y achever leur développement dans des cellules artificielles dont le fond était constitué par une lamelle de verre de telle sorte que les excréments puissent être examinés de façon quantitative en ce qui concerne les kystes amibiens excrétés. Les imagos provenant de ces larves vécurent de 27 à 38 jours; ce temps est suffisant pour permettre à une infection de conduire à la formation de kystes. Cependant les abeilles restèrent libres d'amibes.

4. Les abeilles venant à éclore à partir d'un rayon tiré d'une ruche fortement infectée et maintenu isolé furent mises à l'étuve et conservées en cagette pendant 22 jours. Là non plus on n'a pas pu mẹttre en évidence une infection transmise à l'imago au cours de la vie larvaire.

Compte tenu de ces résultats une infection pré-imaginale doit être considérée comme improbable.

\section{LITERATUR}

BittNer, Alfred, 1966. Beobachtungen über die Amöbenkrankheit der Honigbienen, Dt. Bienenwirtsch. Jg. 17, Nr. 10, S. 284-286.

Lotmar, Ruth, 1945. Die Metamorphose des Bienendarmes, (Apis mell.), Beihefte zur Schweiz. Bienenztg., 1, Heft 10, S. 443-506.

Schulz-Langner, Elisabeth, 1964. Der Entwicklungsgang der in den Malpighischen Gefässen der Honigbiene lebenden Amöbe (Malpighamoeba mellificae PreLL) in Kulturen und im Bienenkörper, Z. f. Bienenforsch., 7, Heft 1, S. 1-22.

Snodgrass, R. E., 1956. Anatomy of the Honey Bee, Cornell University Press, Ithaca, New York.

Anschrift der Verfasserin :

Prof. Dr. Elisabeth Schulz-Langner, Abteilung Bienenkunde des Instituts für Tierzüchtung und Haustiergenetik der Technischen Universität Berlin, 1000 BerLin 33, Lentzeallee 75. 